\title{
A Comprehensive Study on the Effect of Roasting and Frying on Fatty Acids Profiles and Antioxidant Capacity of Almonds, Pine, Cashew, and Pistachio
}

\author{
Hadeel Ali Ghazzawi and Khalid Al-Ismail \\ Department of Nutrition and Food Technology, Faculty of Agriculture, The University of Jordan, Amman 11942, Jordan \\ Correspondence should be addressed to Hadeel Ali Ghazzawi; h.ghazzawi@ju.edu.jo
}

Received 18 September 2017; Revised 2 November 2017; Accepted 29 November 2017; Published 20 December 2017

Academic Editor: Domenico Montesano

Copyright (C) 2017 Hadeel Ali Ghazzawi and Khalid Al-Ismail. This is an open access article distributed under the Creative Commons Attribution License, which permits unrestricted use, distribution, and reproduction in any medium, provided the original work is properly cited.

\begin{abstract}
The aim is to evaluate the effects of frying and roasting on nuts. Frying and roasting were performed according to the local Jordanian home-made cuisine, and the nuts under experiment were raw almonds, pine, cashew, and pistachio. Nuts samples were roasted at $110^{\circ} \mathrm{C}$ for 16 minutes and fried at $175^{\circ} \mathrm{C}$ for 2.5 minutes. The results show that both roasting and frying of nuts did not affect the flavonoids content except for roasted pistachios where significant rise of flavonoids content was detected. Total phenolic content showed no significant differences except for pine nuts in which it increased significantly in both roasting and frying. Oxidative stability, presented by 1,1-diphenyl-2-picryl-hydrazyl (DPPH), was significantly different in all nuts except for pistachio nuts that have shown no differences. Fatty acids profile, presented by saturated fatty acids (SFA), oleic acid (OL), and essential fatty acids (EFA), was affected significantly by roasting and frying, especially for SFA in almonds and pine nuts and $\alpha$-linoleic acid (ALA) contents of pine. In conclusion, the effects of roasting and frying on the aforementioned nuts species were positive for fatty acids profile and antioxidants activity.
\end{abstract}

\section{Introduction}

Consuming nuts is a very common phenomenon across different European countries, with its highest consumption rate in Mediterranean regions [1]. The consumption of nuts (raw or processed) has increased over the last decades due to their availability, reasonable price, and the rise in the nutritional health awareness [2]. The availability of legumes and nuts in Jordan has increased from 40 to $60 \mathrm{~g} /$ person/day. Additionally, the Food Based Dietary Guidelines for Arab Countries in 2013 recommended regular consumption of nuts.

The consumption of nuts yields beneficial effects on health due to their (1) desirable lipid profile, which is higher in unsaturated fatty acids (USFA) than saturated fatty acids (SFA), and (2) high antioxidants contents [3]. Epidemiological and interventional studies have shown how frequent consumption of nuts can enhance cardiovascular health by decreasing serum levels of low-density lipoprotein- (LDL-) cholesterol and risk of developing type II diabetes $[4,5]$.
Expressly that plant-derived omega 3 polyunsaturated fatty acids (PUFA) $\alpha$-linolenic acid (ALA) (which is high in nuts) is deliberated as the precursor of the longer chain fatty acid in the body and hence the eicosapentaenoic acid (EPA) and docosahexaenoic acid (DHA). Clinical trials and prospective studies identified well the last two fatty acids for their beneficial effects on prothrombotic cardiovascular risk factors [6].

Additionally, nuts have also been shown to promote weight maintenance when consumed as the source of fat in moderate diets [7]. This is a healthier practice instead of eating high fat content snacks from unhealthy sources such high energy condense food items (fried food, confectionary snacks, etc.) which are known to contain SFAs and transfatty acids [8]. Oxidation of fat and fat-containing foods is responsible for the deterioration in the food quality and nutritive value. Additionally, the oxidation of PUFA in food may be related to diseases such as atherosclerosis, diabetes, and cancer [9]. 
Moreover, nuts contain certain bioactive compounds which act as antioxidants (such as a variety of phenolic compounds and flavonoids), free radical scavengers, and metal chelates that may also play a role in the reduction of the risk for the development of chronic diseases. Phytochemical compounds are available in almonds [10], pistachio, cashew, and pine nuts. Chen and Blumberg demonstrated that flavonoids and phenols intake can decrease the risk of a number of chronic diseases including cardiovascular diseases, hypercholesterolemia, and diabetes mellitus due to their attributes of being antioxidant and anti-inflammatory and antiproliferation [11]. These antioxidants exist in all parts of the plant; nuts (seeds) are one of the richest sources [12].

Roasting and frying are two of the most common methods of nuts' thermal processing in order to enhance their sensory properties [13-15]. Although there are several commercial products, for example, nut bars or snacks that contain roasted and fried nuts, there is a lack of a scientific comparison of the differences in their nutrients content. Such nutritional alteration might be unfavorable and lead to negative health impacts upon consumption. Roasting and frying not only can change the obvious rawness of nuts but also can subsequently have chemical changes on nuts. More than $50 \%$ of nuts' energy comes from the fat content of nuts [6]; therefore it is important to study the chemical and bioactive changes that take place due to frying and roasting.

In Jordan, one of the common and traditional ways to present the local cuisine (rice-based meals) is to top rice with fried nuts: pine, almonds, peanuts, cashew, and pistachio. Nuts are also introduced as raw, roasted, fried or as a part of desserts in special occasions and ceremonies.

To the authors' best knowledge, there is no work found in the field of analyzing the chemical changing of the raw nuts after roasting or frying according to Jordanian culture. In relevant studies, roasted nuts were tested, but not fried nuts. This study is aimed to investigate the effects of roasting as well as frying on the content of total SFAs, monounsaturated fatty acids (MUFA) presented by oleic acid (OL), essential fatty acids (EFA), oxidative stability presented by 1,1-diphenyl2-picryl-hydrazyl (DPPH), and bioactive compounds presented by flavonoids and phenolic. Roasted and fried nuts which were experimented with in this study were obtained from two major stores in Amman, Jordan.

\section{Materials and Methods}

2.1. Raw and Roasted Nuts. Dehulled raw and commercially roasted nuts (almonds, pine, cashew, and pistachio) (4 kg each) were purchased from two major local nuts stores in Amman. Commercially, the roasting of nuts in Jordan is carried out using a rotating drum roaster at approximately $110^{\circ} \mathrm{C}$ for 16 minutes. The following samples were used for the analysis: Indian cashew (Anacardium occidentale), European pine syn. Scots pine (Pinus sylvestris), pistachio (Pistacia vera "Kerman") from Aleppo, and almond (Prunus amygdalus) from India. Identical cultivar of each type of the raw nuts was roasted in each store to apply similar roasting conditions of the sold one and to assure that the same nuts cultivars are used from the two local nuts stores. The raw and roasted nuts were ground and sieved to obtain fine powder. The samples were then stored at $-20^{\circ} \mathrm{C}$ until they were experimented with in this study.

2.2. Pan Frying of Raw Nuts. Identical raw nuts from each species were used from the two local nuts stores and pan frying of dehulled raw nuts (almonds, pine, cashew, and pistachio) ( $100 \mathrm{~g}$ ) was carried out at $175^{\circ} \mathrm{C}$ for 2.5 minutes using refined corn oil as heating medium. Fried nuts were ground, sieved, and stored at $-20^{\circ} \mathrm{C}$ until they were experimented with in this study. This process was carried out for the four nut types studied in this paper.

2.3. Fat Extraction. About $50 \mathrm{~g}$ of ground raw, roasted, and fried samples (almonds, pine, cashew, and pistachio) were soaked in petroleum ether for about 24 hours and then filtered. The petroleum ether phase was evaporated using a rotary evaporator under vacuum at a temperature not exceeding $40^{\circ} \mathrm{C}$ for 15 minutes. The lipid fraction was stored at $-20^{\circ} \mathrm{C}$ in a freezer for additional analysis.

2.4. Nuts Extraction. The powder of raw, roasted, and fried nuts' (almonds, pine, cashew, and pistachio) extraction was carried out according to the method of [16]: $10 \mathrm{~g}$ of each sample was suspended in $100 \mathrm{ml}$ of $90 \%$ ethanol and continuously shook for 2 hours. After filtration, the samples were vacuum evaporated. The extract was then recovered with $2 \mathrm{ml}$ of $90 \%$ ethanol and assayed for its antioxidant activity, total phenolic compounds, and flavonoids.

2.5. Total Polyphenols Content (TPC). Total polyphenols content was carried out according to the methods of [16]. Samples of each extract $(0.4 \mathrm{ml})$ were mixed with $2 \mathrm{ml}$ of FolinCiocalteu reagent (diluted 10 times). After 3 minutes, $1.6 \mathrm{ml}$ of $7.5 \%$ sodium carbonate was added. The absorbance was read at $750 \mathrm{~nm}$ after 30 minutes of incubation at room temperature $\left(26 \pm 2^{\circ} \mathrm{C}\right)$. Blank sample of gallic acid was used as a reference in spectrophotometric analysis. A standard curve was prepared and the results were expressed as mg gallic acid equivalent/g sample.

2.6. Total Flavonoids Content. $1 \mathrm{ml}$ of the extract was added to $1 \mathrm{ml}$ of aluminum trichloride $(2 \% \mathrm{w} / \mathrm{v})$. After 15 minutes of incubation, the absorbance was measured at $430 \mathrm{~nm}$ and the results were expressed as mg quercetin equivalents per mg sample. A standard curve was prepared by using a blank sample of pure quercetin acid as a reference in spectrophotometric analysis.

2.7. Free Radicals Scavenging Activity (DPPH). The antioxidant activity of the sample extracts was evaluated using 2,2diphenyl-1-1 picrylhydrayl (DPPH) radical according to the method of [17]. An aliquot of $30 \mu \mathrm{l}$ of the sample extracts was added to $0.5 \mathrm{ml}$ of DPPH solution $(25 \mathrm{mg} / \mathrm{l})$ diluted to $5 \mathrm{ml}$ of methanol. A control without extract was also prepared. The mixture was shaken vigorously and allowed to stand for 45 minutes in the dark and the absorbance was measured at 
$515 \mathrm{~nm}$. The antioxidant activity of the extract was calculated using the following formula:

$\%$ Inhibition

$$
\begin{aligned}
= & \frac{\text { Absorbance sample }- \text { Absorbance control }}{\text { Absorbance control }} \\
& * 100 .
\end{aligned}
$$

2.8. Vitamin E Determination. Vitamin E was determined following the method used by [18]. The analysis of vitamin E was carried out using a Knauer HPLC System (Germany). The determination was carried out following the method of Gimeno et al. (2000) [18]. The oil sample of raw, roasted, and fried peanut was diluted in hexane $(1: 10)$. An aliquot of $200 \mu \mathrm{l}$ was transferred to a test tube containing $600 \mu \mathrm{l}$ of methanol and $200 \mu \mathrm{l}$ of the internal standard solution $(300 \mathrm{mg} / \mathrm{ml}$ of $\alpha$-tocopherol acetate in ethanol). The mixtures were mixed, centrifuged at $3000 \mathrm{~g}$ for $5 \mathrm{~min}$, and then filtered through a $0.45 \mathrm{~mm}$ pore size filter. Fifty micrometers was directly injected into the Knaur HPLC system. The mobile phase was methanol-water $(96: 4, \mathrm{v} / \mathrm{v})$ and the elution was performed at a flow rate of $2 \mathrm{ml} / \mathrm{min}$. The analytical column was Venusil XBP, C18 (2) (Agelant Technologies, USA), and was kept at $45^{\circ} \mathrm{C}$. Detection was performed at $292 \mathrm{~nm}$ using Knauer UV detector (model Smartline 2500, Germany).

2.9. Determination of Fatty Acids Profile. Fatty acid methyl esters (FAMEs) of the nuts samples were prepared according to the method outlined in [17]. The prepared FAMEs were studied using capillary Gas Liquid Chromatography (GLC) analysis [19]. The prepared methyl esters were analyzed using capillary GLC column (Restek, Rtx-225, USA, cross-bond 90\%-cyano-propyl-methyl-poly-siloxane, $60 \mathrm{~m}$ and $0.25 \mu \mathrm{m}$ df) immediately after esterification by injection $1 \mu \mathrm{l}$ of the hexane layer through the injection port of the GLC (model GC-2010, Shimadzu, Inc., Kyoto, Japan). The GLC condition was at $15^{\circ} \mathrm{C} / \mathrm{min}$ and held at this temperature for $10 \mathrm{~min}$ utes, and then it was increased from initial column oven temperature $165^{\circ} \mathrm{C}$ to $180^{\circ} \mathrm{C}$ at $1^{\circ} \mathrm{C} /$ minute and then further increased to $220^{\circ} \mathrm{C}$ at $3^{\circ} \mathrm{C} / \mathrm{min}$ and held at this temperature for 10 minutes. Injector temperature was $240^{\circ} \mathrm{C}$ and the flame ionization detector temperature was at $250^{\circ} \mathrm{C}$, flow rate of He was $0.8 \mathrm{ml} / \mathrm{min}$, and the split ratio used was $80: 1$. Fatty acid identification was carried out by ingestion standard fatty acids (Sigma, USA). SFAs were detected as the sum of palmitic acid (C16:0), margaric acid (C17:0), stearic acid (C18:0), arachidic acid (C20:0), and lignoceric acid (C24:0). Monounsaturated fatty acids (MUFA) were detected by OL only as it is the predominant MUFA fatty acids in nuts. PUFA were detected by the sum of essential fatty acids (EFA) alphalinolenic acid LA (an omega-3 fatty acid) and linoleic acid ALA (an omega- 6 fatty acid).

\section{Statistical Analysis}

Each type of nuts from each store was tested separately; then the results altogether were averaged. It is worthy to mention that very close results were obtained from the two stores due to (1) the identical cultivars species that was used by the authors of this paper and because (2) similar conditions of nuts roasting and frying were applied in the two major local nuts stores as well. All tests were done three times and then subsequently averaged. Statistical analyses were performed using SPSS (Statistical Program for Social Sciences) version 17.0 for Windows. All analyses were conducted in triplicate, and data reported as means \pm standard deviation (SD) and differences between means were considered significant at $p$ value $<0.05$ differences between means were considered significant at $p$-value $<0.05$. Fatty acids' area $[\mu \mathrm{V} \cdot \mathrm{s}]$ from the chromatogram was completed and calculated by using Microsoft ${ }^{\circledR}$ Office Excel 2007 (12, 0, 4518, 1014) MSO.

\section{Results and Discussion}

The bioactive compounds of nuts such as the antioxidants capacity, phenols, flavonoids, vitamin E, and fatty acids are associated with numerous health benefits and are affected by heat treatments. Therefore, this study is aimed to investigate the effects of roasting and frying on the content of total saturated fatty acids, essential fatty acids, oxidative stability, and bioactive compounds presented by flavonoids and phenolic acids. Quercetin and gallic acid were used only as standards to expressed total phenolic and total flavonoids contents. Table 1 demonstrates the antioxidant capacity expressed by total flavonoids, total phenolic, and percentage of DPPH inhibition. Our results were not too distant in conclusion to other results from the literature apart from different units were used $[20,21]$.

4.1. Antioxidants Capacity. The antioxidant capacity and high phenol content of nuts whether raw or roasted/fired indicate that the associated health benefits would not be restricted to the lipidic fraction. They also explain that the importance of the stability of phenol compounds preserves from eventual thermal degradation and guarantees their antioxidant potential. Nuts are not a major source of dietary carotenoids [11]. Among the four types of tested nuts, the carotenoids contents were not detected or neglected. Therefore, the majority of the antioxidant capacity in nuts applies on DPPH, flavonoids, and phenolic acids. Table 1 shows that the tested nuts are known for the high content of gallic and quercetin acid which dabble as an antiproliferative, antimutagenic, and antioxidant [20].

Many factors have influenced the levels of phenol compounds in nuts such as the environmental factors, soil composition, and maturation level [20]. Table 1 demonstrates that roasting and frying (i.e., heat treatments) have led to insignificant consequences on total flavonoids among tested raw nuts with the exception of pistachio that showed onefold increase on roasted ones $(\mathrm{raw}=6.71$ and roasted $=13.74$, $p<0.05)$.

This work shows that heat treatments have no significant influence on the total phenolic content expressed by gallic acid in cashew, pistachio, and almonds except for pine which have shown a significant increase on the total phenolic compounds $(p<0.05)$. A similar effect has been also noticed in phenolic acid (gallic acid) for pine nuts. The increase of 
TABLE 1: Effect of roasting and frying on antioxidant activity as compared to raw nuts.

\begin{tabular}{|c|c|c|c|}
\hline & Raw & Roasted & Fried \\
\hline & \multicolumn{3}{|c|}{ Total flavonoids content (mg quercetin acid equivalent/g sample) } \\
\hline Cashew & $4.25 \pm 1.41^{\mathrm{a}}$ & $5.20 \pm 0.34^{\mathrm{a}}$ & $6.94 \pm 2.57^{\mathrm{a}}$ \\
\hline Pistachio & $6.71 \pm 1.17^{\mathrm{a}}$ & $13.74 \pm 1.58^{\mathrm{b}}$ & $6.85 \pm 2.40^{\mathrm{a}}$ \\
\hline Almond & $6.49 \pm 2.43^{\mathrm{a}}$ & $4.58 \pm 1.16^{\mathrm{a}}$ & $10.60 \pm 1.23^{\mathrm{a}}$ \\
\hline \multirow[t]{2}{*}{ Pine } & $4.58 \pm 0.24^{\mathrm{a}}$ & $6.06 \pm 1.03^{\mathrm{a}}$ & $7.95 \pm 0.44^{\mathrm{a}}$ \\
\hline & \multicolumn{3}{|c|}{ Total Phenolic content (mg gallic acid equivalent/g sample) } \\
\hline Cashew & $5.39 \pm 1.88^{\mathrm{a}}$ & $6.73 \pm 0.63^{\mathrm{a}}$ & $7.09 \pm 0.78^{\mathrm{a}}$ \\
\hline Pistachio & $6.74 \pm 1.72^{\mathrm{a}}$ & $10.50 \pm 3.12^{\mathrm{a}}$ & $10.46 \pm 3.45^{\mathrm{a}}$ \\
\hline Almond & $5.87 \pm 1.55^{\mathrm{a}}$ & $8.46 \pm 1.38^{\mathrm{a}}$ & $8.24 \pm 1.85^{\mathrm{a}}$ \\
\hline \multirow[t]{2}{*}{ Pine } & $3.46 \pm 0.35^{\mathrm{a}}$ & $7.62 \pm 2.07^{\mathrm{b}}$ & $6.82 \pm 1.35^{\mathrm{b}}$ \\
\hline & \multicolumn{3}{|c|}{ DPPH Inhibition (\%) } \\
\hline Cashew & $80.87 \pm 8.07^{\mathrm{b}}$ & $59.99 \pm 5.12^{\mathrm{a}}$ & $59.45 \pm 7.71^{\mathrm{a}}$ \\
\hline Pistachio & $78.51 \pm 6.92^{\mathrm{a}}$ & $74.20 \pm 5.61^{\mathrm{a}}$ & $75.60 \pm 7.61^{\mathrm{a}}$ \\
\hline Almond & $59.50 \pm 7.13^{\mathrm{b}}$ & $78.1 \pm 4.61^{\mathrm{b}}$ & $31.64 \pm 3.87^{\mathrm{a}}$ \\
\hline Pine & $48.36 \pm 8.15^{\mathrm{a}}$ & $63.2 \pm 3.32^{\mathrm{ab}}$ & $72.3 \pm 4.82^{\mathrm{b}}$ \\
\hline
\end{tabular}

Values within the same row with different superscript letters are significantly different $(p<0.05)$.

total phenolic content as a result of roasting and frying could be due to the Maillard reaction which results in formation Maillard derivative such as pyrroles and furans that may react with Folin-Ciocalteu reagent [22]. However, roasted nuts have had $14 \%$ more total polyphenols than raw nuts and the percent of free polyphenols decreased [23]. Moreover, studies on almonds and hazelnuts showed an increment in antioxidant activity as an effect of roasting [24, 25].

Nuts' antioxidant capacities were evaluated by the DPPH radical scavenging method, which is based on the measurement of the reduction ability of antioxidants towards the radical DPPH [26]. Free radicals scavenger activity shows different manipulating on different nuts. While heat treatments significantly increase the DPPH activity in pine nuts, such treatments decrease the DPPH activity in cashew nuts $(p<$ $0.05)$. Roasting almonds have increased the DPPH, but frying almonds DPPH was noticed to have decreased. The results indicated that heat treatments significantly increased DPPH values in pine nuts, as opposed to cashews, in comparison to raw ones $(p<0.05)$. Roasting increased DPPH in almonds against the raw peers.

According to Açar and colleagues, roasting nuts may destroy some bioactive compounds, but it can also form antioxidant compounds through the Maillard reaction. However, the total antioxidants capacity after roasting is the result of the thermal degradation of naturally occurring antioxidant compounds and the formation of new Maillard reaction products having antioxidant activity. Chandrasekara and Shahidi suggested that roasting cashews at high temperatureshort time enhances effectively its antioxidant activity [Chandrasekara and Shahidi, 201] which is shown to be true as well in this study.

An increase in those compounds is believed to give the plausible taste in roasted and fried nuts rather than the raw one. Raw nuts are known to have a tart-like taste but once roasted or fried this specific taste can disappear. One of the explanations stems from the increase in flavonoids and phenols.

4.2. Fatty Acids Profile. The literature shows how healthy nuts consumption can be due to their fatty acids composition. Around 62\% of nuts energy is coming from fat [27]. Fatty acids composition of nuts is beneficial because SFA is low and MUFA content is significantly higher than SFA, which has also been demonstrated in this work for specific nut types $(\mathrm{SFA}=$ ranges $7-18 \%$ while MUFA $(\mathrm{OL})$ ranges $40-70 \%$ of total fat). The type of dietary fat intake affects plasma cholesterol level more than the total fat intake [27]. The high content of MUFAs and PUFAs is considered healthy fats in nuts oil content which can counterbalance the unfavorable SFAs [28].

Table 2 demonstrates the effect of roasting and frying on fatty acids profiles expressed by total SFA, MUFA presented by OL, and EFA expressed by (ALA and LA). Heat treatments have shown no alterations on fatty acids for pistachios. This suggests that roasting and frying have no effects on fatty acids profile content in pistachios. Almond nuts' fatty acids were all significantly affected when fried. Almonds' SFA and EFA (ALA and LA) have increased when fried, whereas OL has significantly decreased.

Heat treatments have had a significant effect on pine nuts' fatty acids profile. Our results show that pine nuts' EFA and SFA significantly increased when fried (an explanation might be due to the presence of vegetable oil median used) but as the frying oil median was removed from the nuts surface by blister paper immediately after frying; thus another explanation might be that nuts contain pores during the frying process that might absorb the oil median inside the nuts. On the other hand, roasting pine nuts significantly abridged LA to the half while doubling OL content $(p<$ 
TABLE 2: Effect of roasting and frying on fatty acids profile as compared to raw nuts.

\begin{tabular}{|c|c|c|c|}
\hline & \multicolumn{3}{|c|}{ Fatty acids profile } \\
\hline & Raw & Roasted & Fried \\
\hline & \multicolumn{3}{|c|}{ Saturated fatty acids $\%$} \\
\hline Cashew & $17.83 \pm 0.44^{\mathrm{a}}$ & $18.77 \pm 0.58^{\mathrm{b}}$ & $17.78 \pm 0.45^{\mathrm{a}}$ \\
\hline Pistachio & $11.92 \pm 1.40^{\mathrm{a}}$ & $10.37 \pm 1.11^{\mathrm{a}}$ & $11.27 \pm 0.77^{\mathrm{a}}$ \\
\hline Almond & $7.2 \pm 0.07^{\mathrm{a}}$ & $7.63 \pm 0.18^{\mathrm{b}}$ & $8.15 \pm 0.27^{\mathrm{c}}$ \\
\hline \multirow[t]{2}{*}{ Pine } & $7.09 \pm 0.01^{\mathrm{a}}$ & $7.74 \pm 0.04^{\mathrm{b}}$ & $8.99 \pm 0.06^{c}$ \\
\hline & \multicolumn{3}{|c|}{ Oleic acid\% } \\
\hline Cashew & $60.52 \pm 1.14^{\mathrm{b}}$ & $62.27 \pm 0.89^{\mathrm{b}}$ & $57.18 \pm 0.27^{\mathrm{a}}$ \\
\hline Pistachio & $60.86 \pm 5.14^{\mathrm{a}}$ & $61.55 \pm 1.64^{\mathrm{a}}$ & $61.57 \pm 5.34^{\mathrm{a}}$ \\
\hline Almond & $69.31 \pm 0.18^{\mathrm{b}}$ & $68.37 \pm 0.22^{\mathrm{b}}$ & $65.1 \pm 0.53^{\mathrm{a}}$ \\
\hline \multirow[t]{2}{*}{ Pine } & $39.82 \pm 0.06^{\mathrm{a}}$ & $68.76 \pm 0.5^{\mathrm{b}}$ & $38.29 \pm 0.19^{\mathrm{a}}$ \\
\hline & \multicolumn{3}{|c|}{ Linoleic acid\% } \\
\hline Cashew & $21.51 \pm 0.96^{\mathrm{b}}$ & $18.61 \pm 0.18^{\mathrm{a}}$ & $24.77 \pm 0.50^{\mathrm{c}}$ \\
\hline Pistachio & $26.88 \pm 6.29^{a}$ & $27.90 \pm 0.68^{\mathrm{a}}$ & $26.76 \pm 5.61^{\mathrm{a}}$ \\
\hline Almond & $23.41 \pm 0.28^{\mathrm{a}}$ & $23.94 \pm 0.05^{\mathrm{a}}$ & $26.69 \pm 0.48^{\mathrm{b}}$ \\
\hline \multirow[t]{2}{*}{ Pine } & $52.68 \pm 0.10^{\mathrm{b}}$ & $23.44 \pm 0.54^{\mathrm{a}}$ & $52.16 \pm 0.11^{\mathrm{b}}$ \\
\hline & \multicolumn{3}{|c|}{$\alpha$-linolenic acid $\%$} \\
\hline Cashew & $0.15 \pm 0.01^{\mathrm{a}}$ & $0.35 \pm 0.24^{\mathrm{a}}$ & $0.27 \pm 0.15^{\mathrm{a}}$ \\
\hline Pistachio & $0.33 \pm 0.07^{\mathrm{a}}$ & $0.36 \pm 0.03^{\mathrm{a}}$ & $0.40 \pm 0.06^{\mathrm{a}}$ \\
\hline Almond & $0.052 \pm 0.00^{\mathrm{a}}$ & $0.053 \pm 0.00^{\mathrm{a}}$ & $0.066 \pm 0.01^{b}$ \\
\hline Pine & $0.30 \pm 0.01^{\mathrm{b}}$ & $0.06 \pm 0.00^{\mathrm{a}}$ & $0.56 \pm 0.01^{\mathrm{c}}$ \\
\hline
\end{tabular}

Values within the same row with different superscript letters are significantly different $(p<0.05)$; $\%$ SFA +OL + ALA + LA $=100 \%$.

0.05). Cashew nuts showed no impact in ALA, but LA has significantly increased when fried as well as roasted.

ALA has the proinflammatory actions of the $n-6$ eicosanoids [6]. Therefore, due to the balance of n-6 and n3 PUFA in the diet and its critical effect on cardiovascular disease CVD, the intake of fried almonds and pine should be controlled. The results shown in this work identify how roasted pine nuts can be a better diet balance with respect to the aforementioned health factors. In order to evaluate the oxidative quality of commercial fried nuts, Marmesat and colleagues demonstrated that frying oil changes the composition of fatty acids by the incorporation of substantial contents of the frying fat. High temperature of fat (during frying) may exert some effect on the quality and stability of the oxidative of the oil used in the frying process [6].

The high content of MUFAs and PUFAs is considered healthy fats in nuts oil content which can counterbalance the unfavorable SFAs [28]. Accordingly, nuts have positive impacts as far as CVD is concerned by preventing LDL oxidation, mediated by fatty acids profile and antioxidants content [21].

Figures 1-4 simplified the heat treatments effects among each type of nuts in regard to their fatty acids content. Table 3 summarizes the impacts of heat treatments on fatty acids content among cashew, pistachio, pine, and almonds to simplify the recommendation according to fatty acids type. Table 3 demonstrates ascending ranking of the tested nuts' values which are derived and shown in Table 2. There were no differences recorded in cashew nuts' fatty acid profile when roasted or fried. Cashews are the richest in SFA while

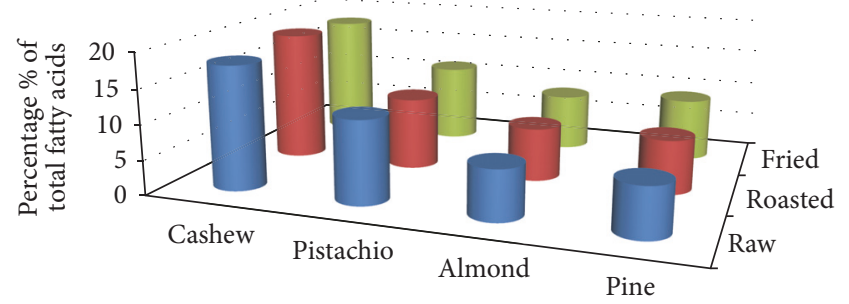

\begin{tabular}{lcccc}
\hline & Cashew & Pistachio & Almond & Pine \\
\hline - Raw & 17.83 & 11.92 & 7.2 & 7.09 \\
- Roasted & 18.77 & 10.37 & 7.63 & 7.74 \\
Fried & 17.78 & 11.27 & 8.15 & 8.99 \\
\hline
\end{tabular}

FIGURE 1: Saturated fatty acids\%.

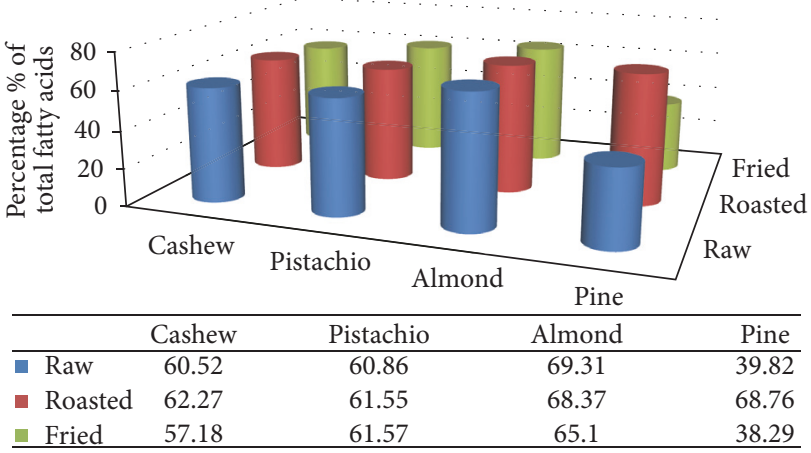

Figure 2: Oleic acid\%. 
TABLE 3: Appraisal between the percentage of fatty acids content among raw and heat-treated nuts.

\begin{tabular}{|c|c|c|c|}
\hline & Raw & Roasted & Fried \\
\hline \multirow{4}{*}{ SFA } & Cashew 17.8\% & Cashew 18.7\% & Cashew 17.8\% \\
\hline & Pistachio 11.9\% & Pistachio $10.4 \%$ & Pistachio 11.3\% \\
\hline & Almonds 7.2\% & Almonds 7.6\% & Almonds $8.1 \%$ \\
\hline & Pine $7.2 \%$ & Pine $7.7 \%$ & Pine $9 \%$ \\
\hline \multirow{4}{*}{$\mathrm{OL}$} & Almond 69.3\% & Pine $68.7 \%$ & Almond 65.1\% \\
\hline & Pistachio $60.9 \%$ & Almond 68.4\% & Pistachio 61.5\% \\
\hline & Cashew 60.5\% & Cashew 62.3\% & Cashew $57.2 \%$ \\
\hline & Pine $40 \%$ & Pistachio 61.5\% & Pine $38.3 \%$ \\
\hline \multirow{4}{*}{ LA } & Pine $52.7 \%$ & Pistachio $27.9 \%$ & Pine $52.2 \%$ \\
\hline & Pistachio 26.9\% & Almond 24\% & Pistachio $26.8 \%$ \\
\hline & Almond 23.4\% & Pine $23.4 \%$ & Almond 26.7\% \\
\hline & Cashew 21.5\% & Cashew $18.6 \%$ & Cashew $24.8 \%$ \\
\hline \multirow{4}{*}{ ALA } & Pistachio $0.33 \%$ & Pistachio $0.36 \%$ & Pine $0.56 \%$ \\
\hline & Pine $0.3 \%$ & Cashew $0.035 \%$ & Pistachio $0.4 \%$ \\
\hline & Cashew 0.15\% & Pine $0.06 \%$ & Cashew $0.27 \%$ \\
\hline & Almond $0.05 \%$ & Almond $0.05 \%$ & Almond $0.06 \%$ \\
\hline
\end{tabular}

$\%$ represents the percentage out of total fat content.

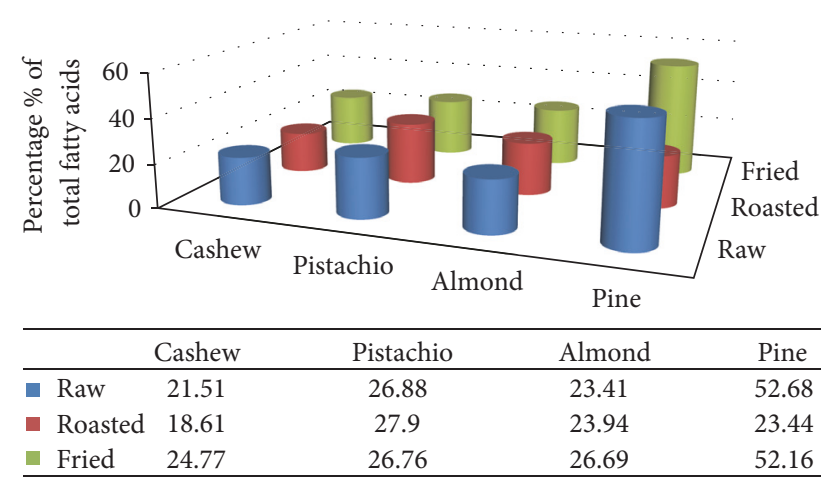

Figure 3: Linoleic acid\%.

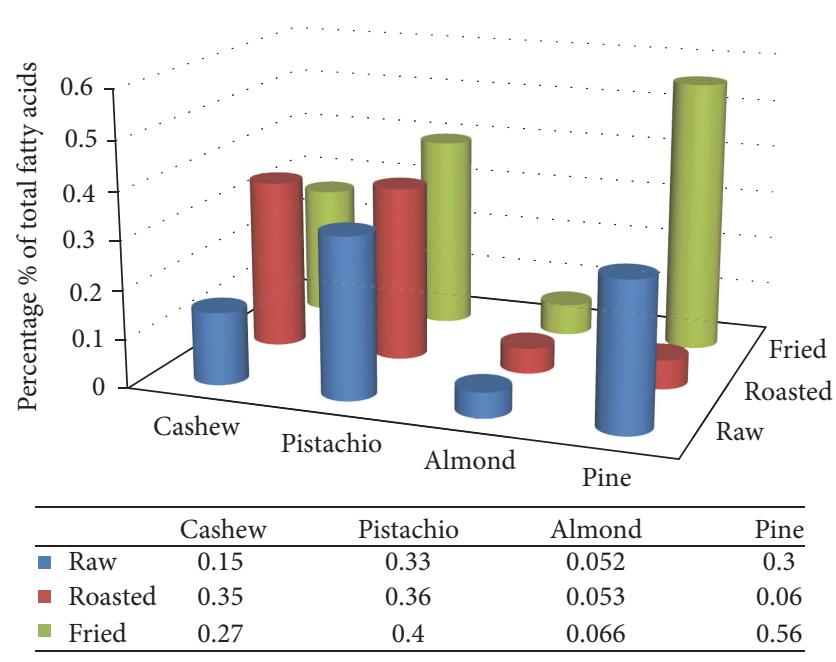

Figure 4: $\alpha$-Linolenic acid\%. almonds and pine are the lowest with near values to each other. Cashews' OL did not differ as previously stated in this section of this work. Raw and fried almonds are the highest in MUFA (expressed by OL), while raw and fried pine nuts were the lowest. Roasted pine nuts were the highest in MUFA content.

In regard to EFA, pine nuts and pistachios have appeared to be the richest sources of it while almonds and cashews were, in contrast, the poorest. The last finding of this paper showed that the heat treatments among cashews and almonds were ineffective with respect to their EFA.

\section{Conclusion}

Nuts generally contain essential nutrients that enhance the antioxidant activity and contribute to health benefits. However, the way of serving, processing, and consuming nuts could affect these nutrients. Indeed, heat-treated nuts (roasted/fried) resulted in a significant positive and/or negative changes of nuts properties, varied according to nuts type. Altogether, heat treatment of nuts could have an impact that cannot be ignored; therefore, we recommend consuming different nuts as raw or as heat-treated in spite of high content of calories as an alternative to nonnutrient high-calories food.

\section{Further Work}

It has been stated that properties of nuts might depend on temperature and time of roasting and frying. This can motivate a future work for conducting similar analysis while taking into consideration roasting/frying temperature, time, and other processing factors. Different effects of roasting/frying 
on various nut species would also be a useful area for further study to have an accurate explanation.

\section{Additional Points}

Research Limitations/Complications. Nuts were brought from local stores that might experience different circumstances with respect to storage/processing factors and the values of the active compounds could be affected.

\section{Conflicts of Interest}

The authors declare no conflicts of interest.

\section{Acknowledgments}

The authors would like to acknowledge the University of Jordan for funding this research study. All the analytic tests were conducted in the laboratories and facilities of the Agriculture College, Nutrition and Food Science Department, during the period of March, 2016-July, 2016.

\section{References}

[1] M. Jenab, J. Sabaté, N. Slimani et al., "Consumption and portion sizes of tree nuts, peanuts and seeds in the European Prospective Investigation into Cancer and Nutrition (EPIC) cohorts from 10 European countries," British Journal of Nutrition, vol. 96, no. 2, pp. 12-23, 2006.

[2] C. E. O’Neil, D. R. Keast, V. L. Fulgoni, and T. A. Nicklas, “Tree nut consumption improves nutrient intake and diet quality in US adults: an analysis of national health and nutrition examination survey (NHANES) 1999-2004," Asia Pacific Journal of Clinical Nutrition, vol. 19, no. 1, pp. 142-150, 2010.

[3] J. Higgs, "The beneficial role of peanuts in the diet - Part 2," Nutrition \& Food Science, vol. 33, no. 2, pp. 56-64, 2003.

[4] C. M. Alper and R. D. Mattes, "Peanut consumption improves indices of cardiovascular disease risk in healthy adults," Journal of the American College of Nutrition, vol. 22, no. 2, pp. 133-141, 2003.

[5] C. M. Alper and R. D. Mattes, "Effects of chronic peanut consumption on energy balance and hedonics," International Journal of Obesity, vol. 26, no. 8, pp. 1129-1137, 2002.

[6] S. Marmesat, J. Velasco, M. V. Ruiz-Méndez, and M. C. Dobarganes, "Oxidative quality of commercial fried nuts: Evaluation of a surface and an internal lipid fraction," Grasas y Aceites, vol. 57, no. 3, pp. 275-283, 2006.

[7] J. Higgs, "The potential role of peanuts in the prevention of obesity," Nutrition \& Food Science, vol. 35, no. 5, pp. 353-358, 2005.

[8] R. J. De Souza, A. Mente, A. Maroleanu et al., "Intake of saturated and trans unsaturated fatty acids and risk of all cause mortality, cardiovascular disease, and type 2 diabetes: Systematic review and meta-analysis of observational studies," BMJ, vol. 351, Article ID h3978, 2015.

[9] I. Elmadfa and M. Kornsteiner, "Fats and fatty acid requirements for adults," Annals of Nutrition and Metabolism, vol. 55, no. 1-3, pp. 56-75, 2009.

[10] P. E. Milbury, C.-Y. Chen, G. G. Dolnikowski, and J. B. Blumberg, "Determination of flavonoids and phenolics and their distribution in almonds," Journal of Agricultural and Food Chemistry, vol. 54, no. 14, pp. 5027-5033, 2006.

[11] C.-Y. O. Chen and J. B. Blumberg, "Phytochemical composition of nuts," Asia Pacific Journal of Clinical Nutrition, vol. 17, no. 1, pp. 329-332, 2008.

[12] K. M. Al-Ismail and T. Aburjai, "Antioxidant activity of water and alcohol extracts of chamomile flowers, anise seeds and dill seeds," Journal of the Science of Food and Agriculture, vol. 84, no. 2, pp. 173-178, 2004.

[13] I. U. Grün, K. Adhikari, C. Li et al., "Changes in the profile of genistein, daidzein, and their conjugates during thermal processing of tofu," Journal of Agricultural and Food Chemistry, vol. 49, no. 6, pp. 2839-2843, 2001.

[14] F. C. Stintzing, M. Hoffmann, and R. Carle, "Thermal degradation kinetics of isoflavone aglycones from soy and red clover," Molecular Nutrition \& Food Research, vol. 50, no. 4-5, pp. 373377, 2006.

[15] H. Rizki, F. Kzaiber, M. Elharfi, S. Ennahli, and H. Hanine, "Effects of roasting temperature and time on the physicochemical properties of sesame (Sesamumindicum. L) seeds," International Journal of Innovation and Applied Studies, vol. 11, pp. 148-156, 2015.

[16] T. Sun and C.-T. Ho, "Antioxidant activities of buckwheat extracts," Food Chemistry, vol. 90, no. 4, pp. 743-749, 2005.

[17] S. W. Christopherson and R. L. Glass, "Preparation of milk fat methyl esters by alcoholysis in an essentially nonalcoholic solution," Journal of Dairy Science, vol. 52, no. 8, pp. 1289-1290, 1969.

[18] E. Gimeno, A. I. Castellote, R. M. Lamuela-Raventós, M. C. de la Torre, and M. C. López-Sabater, "Rapid determination of vitamin $\mathrm{E}$ in vegetable oils by reversed-phase high-performance liquid chromatography," Journal of Chromatography A, vol. 881, no. 1-2, pp. 251-254, 2000.

[19] K. Yanagimoto, K.-G. Lee, H. Ochi, and T. Shibamoto, "Antioxidative activity of heterocyclic compounds found in coffee volatiles produced by Maillard reaction," Journal of Agricultural and Food Chemistry, vol. 50, no. 19, pp. 5480-5484, 2002.

[20] L. T. Abe, F. M. Lajolo, and M. I. Genovese, "Genovese Maria Inés: Comparison of phenol content and antioxidant capacity of nuts," Ciência e Tecnologia de Alimentos, vol. 30, no. 1, pp. 254-259, 2010.

[21] J. M. Harnly, R. F. Doherty, G. R. Beecher et al., "Flavonoid content of U.S. fruits, vegetables, and nuts," Journal of Agricultural and Food Chemistry, vol. 54, no. 26, pp. 9966-9977, 2006.

[22] J. A. Vinson and Y. Cai, "Nuts, especially walnuts, have both antioxidant quantity and efficacy and exhibit significant potential health benefits," Food \& Function, vol. 3, no. 2, pp. 134-140, 2012.

[23] V. Schmitzer, A. Slatnar, R. Veberic, F. Stampar, and A. Solar, "Roasting Affects Phenolic Composition and Antioxidative Activity of Hazelnuts (Corylus avellana L.)," Journal of Food Science, vol. 76, no. 1, pp. S14-S19, 2011.

[24] I. Garrido, M. Monagas, C. Gómez-Cordovés, and B. Bartolomé, "Polyphenols and antioxidant properties of almond skins: Influence of industrial processing," Journal of Food Science, vol. 73, no. 2, pp. C106-C115, 2008.

[25] M. J. Jung, S.-I. Heo, and M.-H. Wang, "Free radical scavenging and total phenolic contents from methanolic extracts of Ulmus davidiana," Food Chemistry, vol. 108, no. 2, pp. 482-487, 2008.

[26] Ö. C. Açar, V. Gökmen, N. Pellegrini, and V. Fogliano, "Direct evaluation of the total antioxidant capacity of raw and roasted 
pulses, nuts and seeds," European Food Research and Technology, vol. 229, no. 6, pp. 961-969, 2009.

[27] M. Sharma, S. Khurana P, and R. Kansal, "Choosing quality oil for good health and long life," Indian Journal of Health and Wellbeing, vol. 7, pp. 254-261, 2016.

[28] E. Ros and J. Mataix, "Fatty acid composition of nuts - Implications for cardiovascular health," British Journal of Nutrition, vol. 96, no. 2, pp. S29-S35, 2006. 

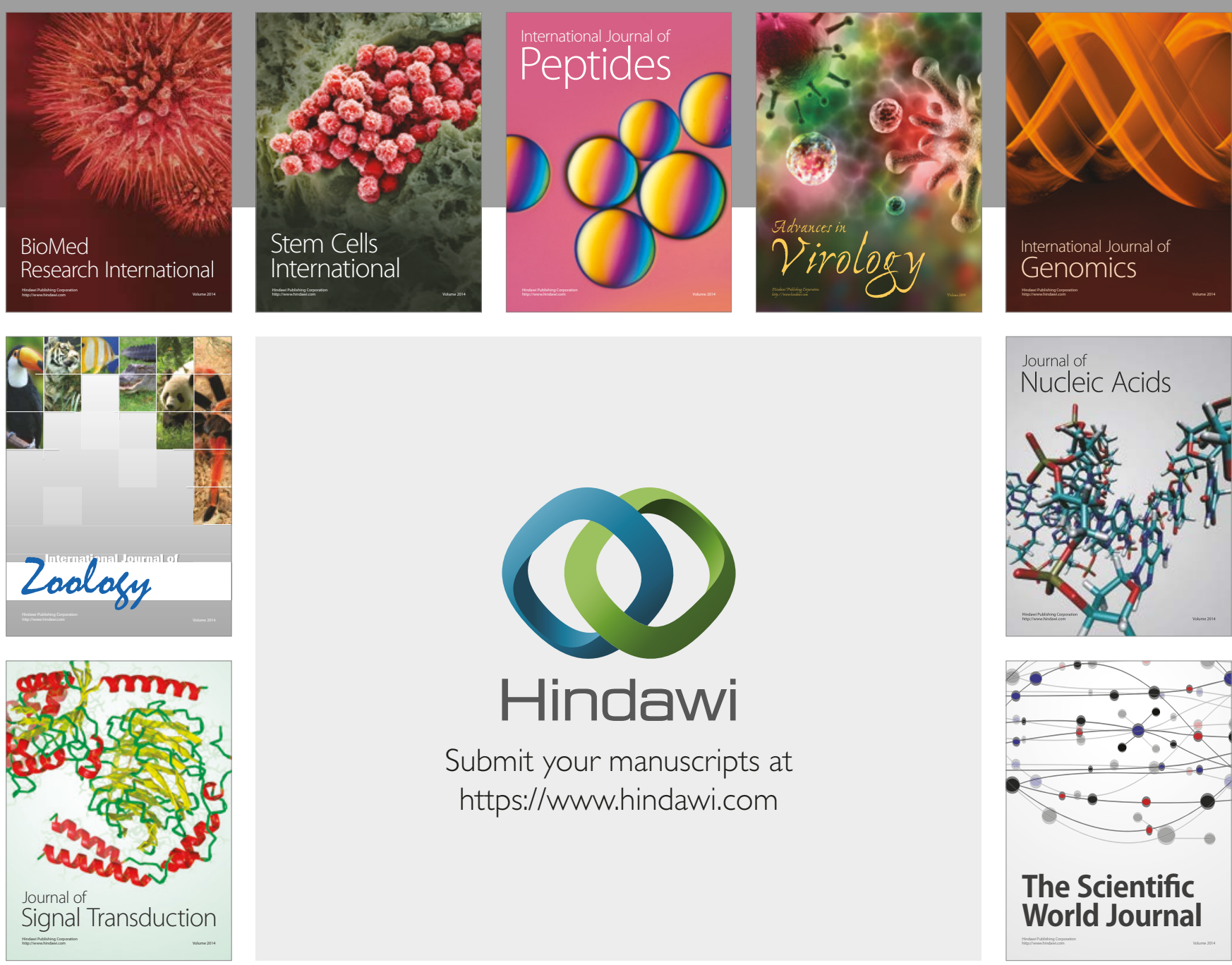

Submit your manuscripts at

https://www.hindawi.com
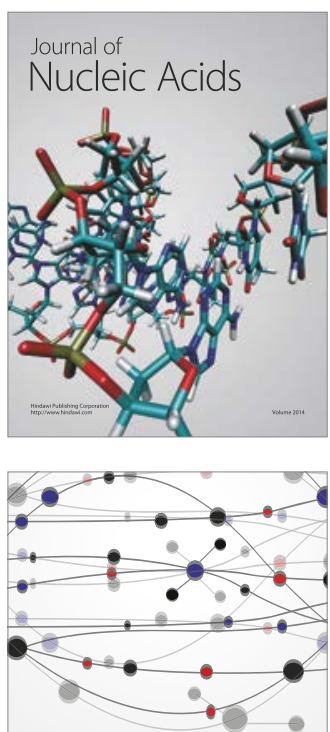

The Scientific World Journal

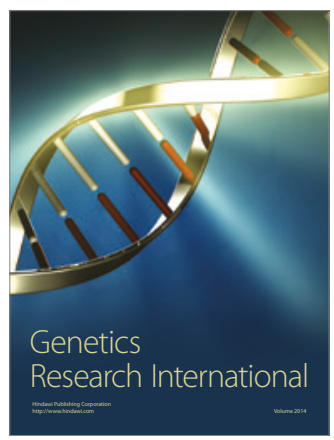

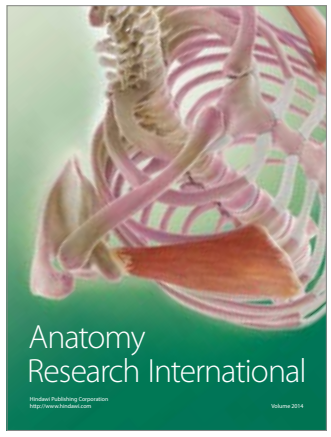

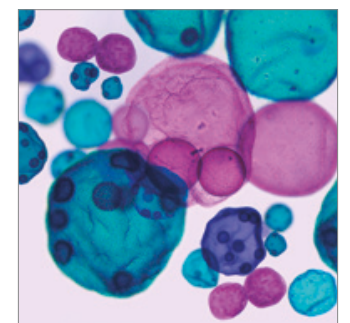

International Journal of Microbiology
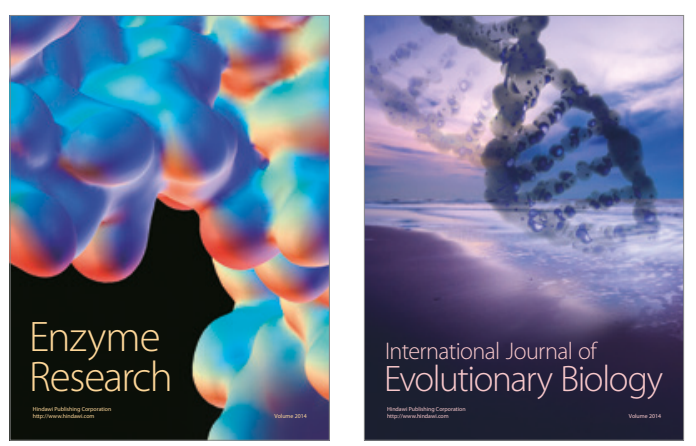
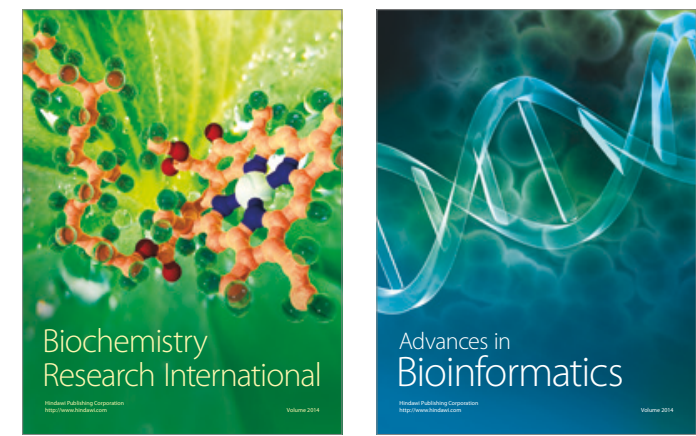

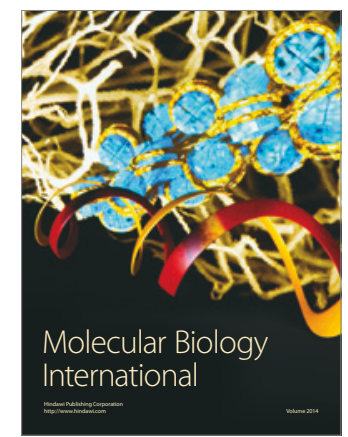

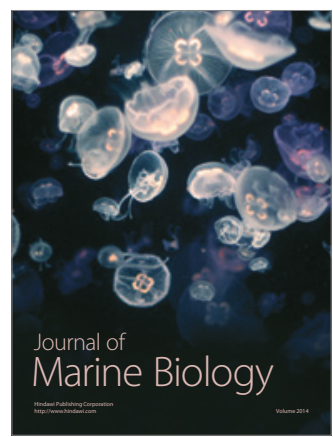

